\title{
A Standard FODO Lattice with Adjustable Momentum Compaction *
} RECEIVED

\author{
Brookhaven National Laboratory, Upton, NY, 11973, USA
}

D. Trbojevic and E. Courant

\section{Abstract}

An existing lattice made of identical FODO cells can be modified to have adjustable momentum compaction. The modified lattice consists of repeating superperiods of four FODO cells where every two cells have different horizontal phase advance. In existing FODO cell rings an additional quad bus is required for every two consecutive cells. This allows tuning of the momentum compaction or $\gamma_{t}$ (transition) to any desired value. A value of the $\gamma_{t}$ could be an imaginary number. A drawback of this modification is relatively large values of the dispersion function (two or three times larger than in the regular FODO cell design).

\section{INTRODUCTION}

Particles travel along the reference orbit in an accelerator ring with momentum $p_{0}$ and period of revolution $T_{0}$. If they have a momentum deviation $\Delta p$, the time of the arrival a the point of observation will be different. An offset in the revolution period $\Delta T$ is given by:

$$
\frac{\Delta T}{T_{0}}=\left(\alpha-\frac{1}{\gamma^{2}}\right) \frac{\Delta p}{p_{0}}
$$

where $\alpha$, the em momentum compaction is a property of the lattice, and $\eta=\alpha-\gamma^{-2}$ is called the em phase-slip factor; $\gamma$ is the Lorentz relativistic factor for the on-momentum particle. The momentum compaction factor is a measure of the path length difference between the off-momentum particle and the on-momentum particle. The transition energy $\gamma_{t}$ is the energy at which $\eta$ vanishes, i.e. it equals $1 / \sqrt{\alpha}$. In many accelerators $\gamma_{t}$ lies in the acceleration range. We shall show that an existing FODO lattice can be modified so as to make $\gamma_{t}$ either very large or even imaginary (negative $\alpha$ ). This could be used to for example avoid having to cross transition, or to make zero momentum compaction isochronous storage rings.

The momentum compaction of a lattice, to the first order, is an integral of the dispersion function $D$ through the dipoles:

$$
\alpha=\frac{1}{C_{0}} \oint \frac{D(s)}{\rho(s)} d s,
$$

where $\rho$ is the radius of curvature and $s$ is the longitudinal path length measured along the reference orbit with a circumference $C_{0}$. There are many ways to devise an accelerator lattice with either fixed or adjustable value of the momentum compaction [1],[3],[4],[5]. Vladimirski and Tarasov [1] propose use of reverse bend dipoles to make the momentum compaction negative. Teng [6] shows that a

\footnotetext{
- Work performed under the auspices of the U.S. Department of Energy
}

straight section with a phase advance of $\pi$ can makethe dis persion closed orbit negative at dipoles. Iliev [3] and Guignard [4] use a harmonic approach, where the betatron function is modulated to produce negative values of the momentum compaction by way of resonance conditions. We have reported earlier [5] and [8] the use of flexible-momentum compaction lattices to minimize dispersion values.

\section{NORMALIZED DISPERSION FUNCTION}

The dispersion function $D$ needs to be adjusted through the FODO cell to obtain a different integral of its values through dipoles. Because the dispersion function satisfies a second order inhomogeneous differential equation of motion [7] it is suitable to use the normalized dispersion function with components $\xi$ and $\chi$ as previously defined [5]:

$$
\xi=\sqrt{\beta_{x}} D^{\prime}-\frac{\beta_{x}^{\prime}}{2 \sqrt{\beta_{x}}} D, \quad \chi=\frac{1}{\sqrt{\beta_{x}}} D
$$

where $\beta_{x}$ and $\beta_{x}^{\prime}$ are respectively the horizontal betatron amplitude function and its derivative [7], $\xi$ and $\chi$ projections of the normalized dispersion vector, and $\phi$ is identical to the horizontal Floquet betatron phase advance in the region where there is no dipole. An example of the standard

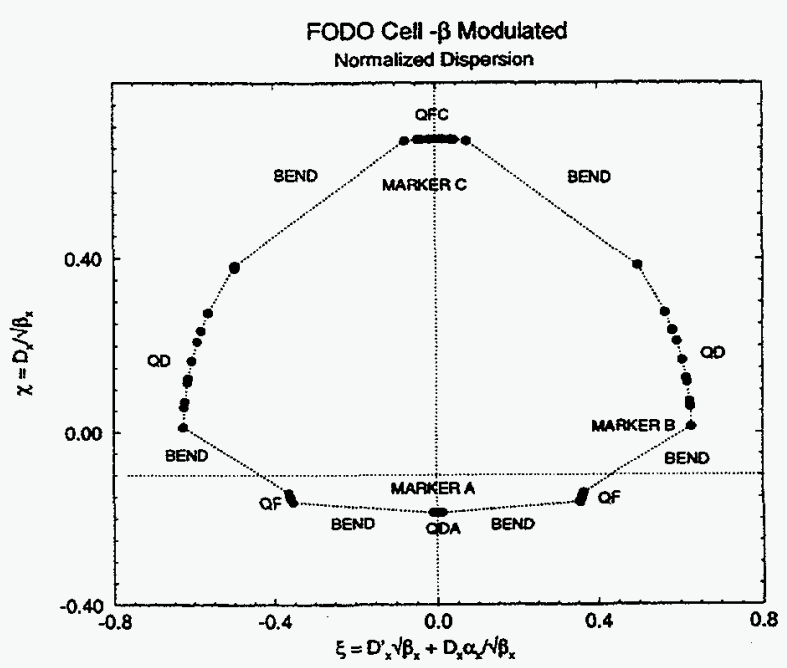

Figure 1: Normalized Dispersion function within the $\beta$ modulated three FODO cells.

three FODO cell lattice design is chosen from the existing Relativistic Heavy Ion Collider (RHIC) at Brookhaven National Laboratory, where the dipole length is $L_{d}=9.45 \mathrm{~m}$ and the cell length $L_{\varepsilon} \simeq 29.6 \mathrm{~m}$. 
Figure 1 shows the normalized dispersion within the three FODO cell lattice with quadrupoles set for an imaginary $\gamma_{t}$ condition.

\section{FODO CELLS WITH ADJUSTABLE MOMENTUM COMPACTION}

The three FODO cells example has the same number of cells as it had previously been selected by Guignard [4]. We chose the point of reflective symmetry of all CourantSnyder functions at the middle of the second FODO cell. The momentum compaction will have a negative value for all three cells if it is negative in left part of the point of symmetry. A negative value of the dispersion function through the first two dipoles should overcome a positive value through the third dipole. The slope of CourantSnyder functions at the beginning and at the point of reflective symmetry, should be zero. A condition of the negative dispersion at beginning of FODO cells is presented in the normalized dispersion plot by the point below the origin $A$, as shown in figure 1. A positive bend by an angle $\theta$ of a dipole is presented by a vector parallel to the $\xi$ axis pointing always towards to positive $\xi$ axis in the normalized dispersion coordinates. Courant-Snyder functions in the the quad perturbed case is shown in figure 2 . The first two dipoles

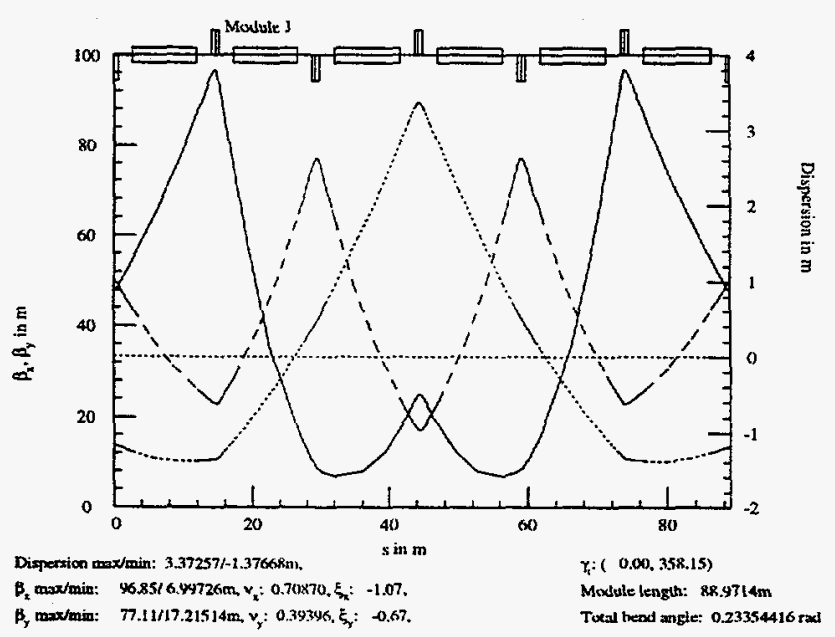

Figure 2: Courant-Snyder functions within the three $\beta$ perturbed cells.

in this example produce a large horizontal offset along the $\xi$ axis in the normalized dispersion plot (up to the marker $B$ in figure 2). A requirement to have zero slopes of the dispersion and amplitude functions at the point of reflective symmetry puts a strong demand on the focusing quadrupole $C$. In a standard FODO cell the horizontal betatron phase per cell of $\pi / 2$ or $90^{\circ}$ provides the smallest value for the dispersion function. In the RHIC FODO cell the maximum dispersion is equal to $D_{\max }=1.8 \mathrm{~m}$ for the cell tune of $\nu_{x} \simeq 81^{\circ}$. The quadrupoles strengths which made the momentum compaction to be negative (or $\gamma_{t}=i 358$ ) are:

\begin{tabular}{||c|c||}
\hline \hline Q. Strengths & $\kappa(1 / \mathrm{m})$ \\
\hline$\kappa_{Q D A}$ & 0.05648 \\
$\kappa_{Q F}$ & 0.07330 \\
$\kappa_{Q D}$ & 0.09634 \\
$\kappa_{Q F C}$ & 0.13057 \\
\hline
\end{tabular}

An attempt to create an example of a row of FODO cells with a different reflective symmetry point which would be in the middle of the four cells, as it was previously reported (see [3]), resulted in much stronger disturbance of the betatron functions. The transition energy is $\gamma_{t}=17.8$ for the three FODO cell under normal operating conditions, while the $\gamma_{t}$ value for one of the quadrupole perturbed cases is $\gamma_{t}=i 358.15$ (as presented in fig. 2). The maximum and minimum value of the dispersion for the imaginary $\gamma_{t}$ result presented in Figure $2 D_{\max }=3.37 \mathrm{~m}$ and $D_{\min }=-1.38 \mathrm{~m}$ which is twice larger than dispersion values when the FODO cells operate at optimum tune $(\nu \sim \pi / 2)$.

\section{CONCLUSION}

The momentum compaction of the lattice made of the standard FODO cells could be adjusted by the modulation of the betatron function to any desired value with a drawback of larger values of the dispersion and betatron amplitude function. As it was previously reported [4], [3], a perturbation of the betatron amplitude function by the existing FODO cell quadrupoles produced any value of the momentum compaction. A range of dispersion function offsets with this perturbation was within twice of the optimum FODO cell dispersion values. The maximum values of the $\beta_{x}$ and $\beta_{y}$ are less than twice of the value at optimum betatron tunes $(\nu=\pi / 2)$. The beam size was less than $\sqrt{2}$ larger due to the amplitude function perturbation. In the presented report a resonance condition was not necessary to achieve different values of the momentum compaction within standard FODO cells, which was a necessary condition in previous reports [3] [4]. We used an existing FODO lattice to accommodate the momentum compaction value, but we do not recommend it for a new lattice design.

\section{REFERENCES}

[1] V.V. Vladimirski and E.K. Tarasov, " Theoretical Problems of the Ring Accelerators", USSR Academy of Sciences, Moscow (1955).

[2] T. Riesselada, Design of Quadrupole schemes to modify Gamma Transition, CERN PS/90-51 (1990).

[3] A.I. Yliev, "Analytic Approach to Design of High Transition Energy Lattice with Modulated $\beta$ Function", Proc. of 1991 IEEE Part. Accel. Conf., 1991, San Francisco, California, pp 1907-1909.

[4] G. Guignard, "A Lattice with no Transition and Largest Aperture", Proc. of 1989 IEEE Part. Accel. Conf., March 20-23, 1989, Chicago, IL, pp 915-917.

[5] D. Trbojevic, D. Finley, R. Gerig, and S. Holmes, "Design Method for High Energy Accelerator without Transition En- 
ergy", Proc. of Second European Particle Accelerator Conference, Nice, France, June 1990, pp 1536-1538.

[6] L.C. Teng, "Infinite Transition-energy Synchrotron Lattice using $\pi$-straight Section”, Part. Acc. 4, pp. 81-85 (1972);

[7] E.D. Courant and H.S. Snyder, "Theory of the Alternating Gradient Synchrotron", Ann. Phys. 3, 1 (1958).

[8] S. Y. Lee, K.Y. Ng, D. Trbojevic, "Minimizing dispersion in flexible-momentum compaction lattices", Physical Review E, Vol. 48, Number 4, October 1993, pp. 3040-3048.

\section{DISCLAIMER}

This report was prepared as an account of work sponsored by an agency of the United States Government. Neither the United States Government nor any agency thereof, nor any of their empleyees, makes any warranty, express or implied, or assumes any legal liability or responsibility for the accuracy, completeness, or usefulness of any information, apparatus, product, or process disclosed, or represents that its use would not infringe privately owned rights. Reference herein to any specific commercial product, process, or service by trade name, trademark, manufacturer, or otherwise does not necessarily constitute or imply its endorsement, recommendation, or favoring by the United States Government or any agency thereof. The views and opinions of authors expressed herein do not necessarily state or reflect those of the United States Government or any agency thereof. 


\section{DECCLAMER}

Portions of this document may be illegible in electronic image products. Images are produced from the best available original document. 